\section{Enfermedad de Crohn e infección de prótesis de cadera por fístula enteral}

\section{Sr. Director:}

Presentamos un caso de infección prótesis articular a los pocos meses de su implantación.

Mujer de 37 años con antecedentes de luxación congénita de caderas, enfermedad de Crohn desde los 17 años con afectación ileocecal que cursaba con brotes frecuentes y obesidad. A los 31 años fue sometida a colectomía subtotal y a anastomosis ileorrectal. Posteriormente presentó insuficiencia suprarrenal secundaria que trataba con hidrocortisona $30 \mathrm{mg}$ al día. En julio del 2004 le fue insertada una prótesis total de cadera derecha $\left(\right.$ Zimmer $\left.^{\circledR}\right)$ por dolor incapacitante. La intervención duró 190 minutos y recibió cefazolina $2 \mathrm{~g}$ en dosis única como profilaxis quirúrgica. En el postoperatorio hubo hemorragia local importante. Durante los 4 días posteriores presentó diarrea líquida sin productos patológicos. Tres meses después acudió por fiebre elevada $\left(39,5^{\circ} \mathrm{C}\right)$, escalofríos, dolor en cadera derecha y supuración purulenta en la proximidad de la a través de la cicatriz. En la analítica destacaba 25.800 leucocitos $/ \mathrm{mm}^{3}$ (82\% segmentados, $8 \%$ cayados) hemoglobina de $10,3 \mathrm{mg} / \mathrm{dl}$ con VCM de $71 \mathrm{fl}$ y 851.000 plaquetas $/ \mathrm{mm}^{3}$ e hipoalbuminemia $(2,1 \mathrm{~g} / \mathrm{dl})$, dos hemocultivos fueron negativos. La radiografía mostraba aumento de partes blandas y aire en la proximidad de la prótesis (Fig. 1). La paciente fue intervenida de urgencia comprobando la existencia de una fístula desde la piel a la región cercana a la prótesis donde se situaba una colección de material purulento y esfacelos que fue drenada. En el cultivo del absceso se aislaron Escherichia coli, Proteus mirabillis y Enterococcus faecium. Se inició tratamiento parenteral con vancomicina $1 \mathrm{~g} / 12$ horas e imipenem $500 \mathrm{mg} / 6$ horas y curas en el quirófano. El quinto día se realizó una TAC abdominal que evidenció varios trayectos fistulosos que se originaban en el ileon de escasos cm de longitud pero sin demostrarse conexión con el área cercana a la prótesis. Mediante una laparotomía se objetivó un trayecto fistuloso desde un asa de ileon hasta la proximidad de la cadera derecha. Se resecaron $20 \mathrm{~cm}$ de intestino delgado afecto y se practicó una ileostomía. En el examen anatomopatológico se encontraron signos de actividad de enfermedad de Crohn y se confirmó la existencia de un fragmento de tracto fistuloso. La paciente fue dada de alta con tratamiento oral levofloxacino 500 $\mathrm{mg} / 24$ horas y cefuroxima $500 \mathrm{mg} / 12$ horas de modo indefinido posponiéndose la retirada de la prótesis.

La infección de una prótesis de cadera debida a fístulas de origen digestivo es una complicación muy infrecuente (1-3). Suelen aparecer en pacientes de edad avanzada y en el contexto de una enfermedad neoplásica colorrectal $(3,4)$. La radioterapia, quimioterapia y el tratamiento con glucocorticoides pueden incrementar el riesgo de padecer esta complicación (3,5). Aunque la mayoría de los casos de fístulas entre el tracto digestivo y la articulación de la cadera se originan en el intestino grueso $(1,5)$, hay casos cuyo origen es el intestino delgado (6), como en nuestro caso. Hay casos de enfermedad de Crohn que han desarrollado una fístula hasta la cadera después de la formación un absceso del psoas (7).

La relación temporal entre la inserción protésica y la aparición de la fístula es muy variable, en la mayoría de los casos aparece a los pocos años de la misma (5) aunque en un caso apareció 27 años después de la inserción de la prótesis (4) y en otros, como el que presentamos, la fistulización se produjo a los pocos meses de colocar la prótesis $(3,8)$.

La mortalidad de las infecciones prótesicas articulares por fístulas digestivas es muy elevada (50\% aproximadamente) y suele acontecer en ancianos con pluripatología $(1,3,5)$. En los casos de

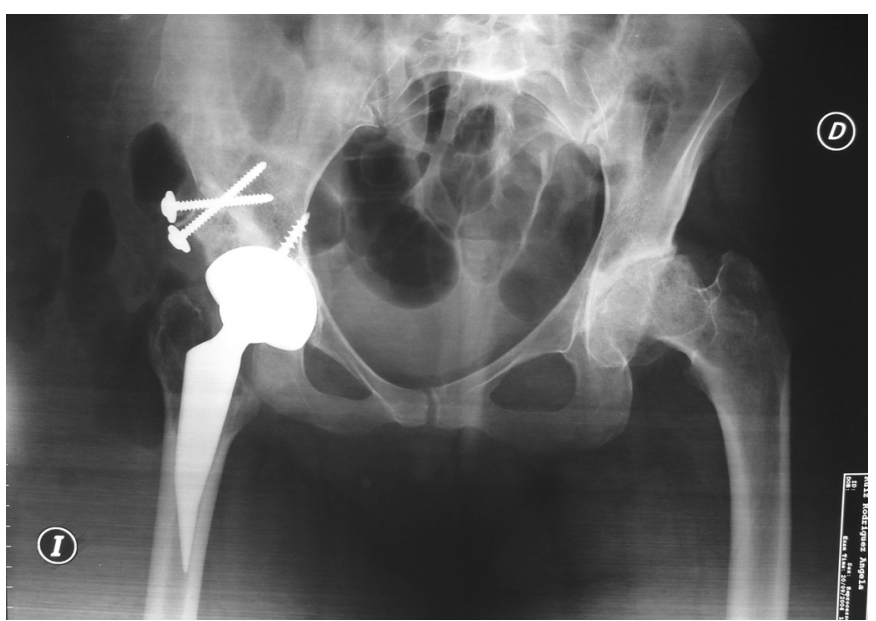

Fig. 1. Radiografía de caderas previa a la cirugía con colecciones aéreas en proximidad de prótesis de cadera y región glútea.

enfermedad de Crohn y fístula que alcanza la articulación de la cadera la mortalidad es menor (7), especialmente cuando la enfermedad es conocida antes de producirse la infección articular (9).

Aunque hay algún caso con buena evolución con antibióterapia prolongada y tratamiento quirúrgico de la fístula, pero sin la retirada de la prótesis (3), el grado de contaminación y la existencia de material protésico obliga a la retirada de la prótesis en la mayoría de los casos (5). En el presente caso se ha mantenido un tratamiento antibiótico supresor crónico vigilando la evolución de la paciente debido al elevado riesgo de recidiva de la infección, que muy probablemente condicionará la retirada de la prótesis en los próximos meses.

\section{A. Ramos Martínez, M. J. García Navarro, E. Muñez Rubio, J. Jiménez Cristóbal ${ }^{1}$, P. Torrijos Garrido ${ }^{1}$}

Unidad de Infecciosas. Servicio de Medicina Interna. Servicio de Traumatología ${ }^{I}$. Hospital Universitario Puerta de Hierro. Universidad Autónoma. Madrid

1. Gómez J, Rodríguez M, Baños V, et al. Infección de prótesis articulares: epidemiología y clínica. Estudio prospectivo 1992-1999. Enferm Infecc Microbiol Clin 2002; 20: 74-7.

2. Gillespie WJ. Prevention and management of infection after total joint replacement. Clin Infect Dis 1997; 25: 1310-7.

3. Thompson NW, Swinson BD, Wilson DS, et al. Hip arthroplasty complicated by colo articular sinus formation- a case report. Acta Orthop Scand 2002; 73: 601-3.

4. Bach CM, Nogler M, Wimmer C, et al. Fistula between a total hip arthroplasty and the rectum. Clin Orthop Rel Res 2001, 388: 143-6.

5. Levin JS, Rodriguez AA, Luong K. Fistula between the hip and the sigmoid colon after total hip arthroplasty. J Bone Joint Surg Am 1997; 79 : 1240-2.

6. Linscheid RL, Kelly PJ, Symmonds RE. Emphysematous cellulitis of the hip and thigh resulting from enteric fistula. J Bone Joint Surg Am 1963; 45: 1691-7.

7. Ogihara M, Masaki T, Watanabe T, et al. Psoas abscess complicating Crohn's disease: report of a case. Surg Today 2000; 30: 759-63.

8. Gabrion A, Mertl P, Gaullier O, et al. Uretero-acetabular fistula after removal of a septic total hip prosthesis. Rev Chir Orthop Reparatrice Appar Mot 1999; 85: 735-9.

9. Shreeve DR, Ormerod LP, Dunbar EM. Crohn's disease with fistulae involving joints. R Soc Med 1982; 75: 946-8. 